\title{
De-escalation of Prasugrel Results in Higher Percentage of Patients within Optimal Range of Platelet Reactivity: Analysis from the HOST- REDUCE-POLYTECH-ACS Trial
}

\author{
Hak Seung Lee ${ }^{1}$ Kyung Woo Park ${ }^{1}$ Jeehoon Kang \\ ${ }^{1}$ Department of Internal Medicine, Cardiovascular Center, Seoul
National University Hospital, Seoul, Republic of Korea \\ Thromb Haemost 2021;122:160-162.
}

\author{
Jung-Kyu Han ${ }^{1}$ Hyo-Soo Kim ${ }^{1}$
}

Address for correspondence Kyung Woo Park, MD, PhD, MBA, Department of Internal Medicine, Cardiovascular Center, Seoul National University Hospital, 101 Daehak-ro, Jongro-gu, Seoul 03080, Korea (e-mail: kwparkmd@snu.ac.kr).
Obtaining and maintaining the optimal balance between ischemic and bleeding risk is a key objective during antiplatelet therapy. Although the current guidelines do not support routine platelet function testing (PFT) to guide antiplatelet therapy, assessment of platelet function may help identify suboptimal- (high on-treatment platelet reactivity [PR], HPR) or over-inhibition (low on-treatment PR: LPR) of platelets. ${ }^{1,2}$ We recently reported the results of a large-scale randomized clinical trial where prasugrel-based de-escalation significantly reduced net adverse clinical events (a composite of ischemic outcomes and bleeding outcomes). ${ }^{3}$ The major benefit of de-escalation over conventional-dose prasugrel was a significant reduction in bleeding without any increase in ischemia. However, it remains to be seen whether such clinical results can be corroborated by pharmacodynamic data.

This study is a preplanned analysis from the HOST-REDUCE-POLYTECH-ACS trial, an investigator-initiated, randomized, parallel-group, open-label, adjudicator-blinded, multicenter trial done at 35 study sites in Korea. ${ }^{3}$ Enrolled patients with acute coronary syndrome (ACS) receiving percutaneous coronary intervention $(\mathrm{PCI})$ meeting the core indication for prasugrel were randomly assigned to either the de-escalation or conventional group. After 1 month of treatment with $10 \mathrm{mg}$ prasugrel plus $100 \mathrm{mg}$ aspirin daily, the deescalation group received $5 \mathrm{mg}$ prasugrel, while the conventional group continued to receive $10 \mathrm{mg}$. All patients gave informed consent. The study was approved by the institutional ethics committee of each participating site (NCT02193971).

PFT was done using the VerifyNow (Accriva Diagnostics, San Diego, California, United States) point-of-care P2Y 12 function assay. Blood samples were obtained from patients in the fasting state. Based on previous studies, PR unit (PRU) $\leq 85$ was defined as LPR, $85<$ PRU $\leq 208$ as OPR (optimal ontreatment PR), and PRU $>208$ as $\mathrm{HPR}^{2,4,5}$ The primary analysis endpoint was the percentage of patients within the therapeutic range or OPR during administration of allocated therapy. Secondary endpoints included the percentage of patients with LPR and the mean PRU of each allocated group.

PFT was performed in 163 of 2,338 patients enrolled in the prasugrel randomization arm (81 patients vs. 82 patients in the de-escalation vs. conventional group, respectively). PFT was assessed at 1-month follow-up before prasugrel deescalation, and during 1-year follow-up while on either the $5 \mathrm{mg}$ de-escalated dose or the $10 \mathrm{mg}$ conventional dose. Overall, the mean age of patients was 59 years. A total of $86.5 \%$ patients were men, $36.8 \%$ had diabetes mellitus, and $27.0 \%$ were smokers. The baseline characteristics are shown in -Supplementary Table $\mathbf{S 1}$ (available in the online version). Similar to the results of the main study, the study population was predominantly males, the mean age was 59 , and those with diabetes, hypertension, and dyslipidemia were approximately 37,60 , and $77 \%$, respectively. There were no significant differences in the baseline clinical characteristics and mode of presentation between the two groups. Regarding the number of clinical events in this group, the number of ischemic events was similar ( 5 vs. 5 for conventional vs. deescalation group), while that of bleeding was numerically lower but not statistically different ( 3 vs. 1 for conventional vs. de-escalation group). PRU values plotted according to treatment are shown in - Fig. 1A. At 1 month, there was no difference between the de-escalation group and the conventional group (PRU presented as median with 95\% confidence interval [CI], 56 [57-80] vs. 63 [60-89], $p=0.55$ ). However, received

December 18, 2020

accepted

April 27, 2021

published online

April 30, 2021 (c) 2021. Thieme. All rights reserved. Georg Thieme Verlag KG,

Rüdigerstraße 14,

70469 Stuttgart, Germany
DOI https://doi.org/ 10.1055/a-1496-7987. ISSN 0340-6245. 

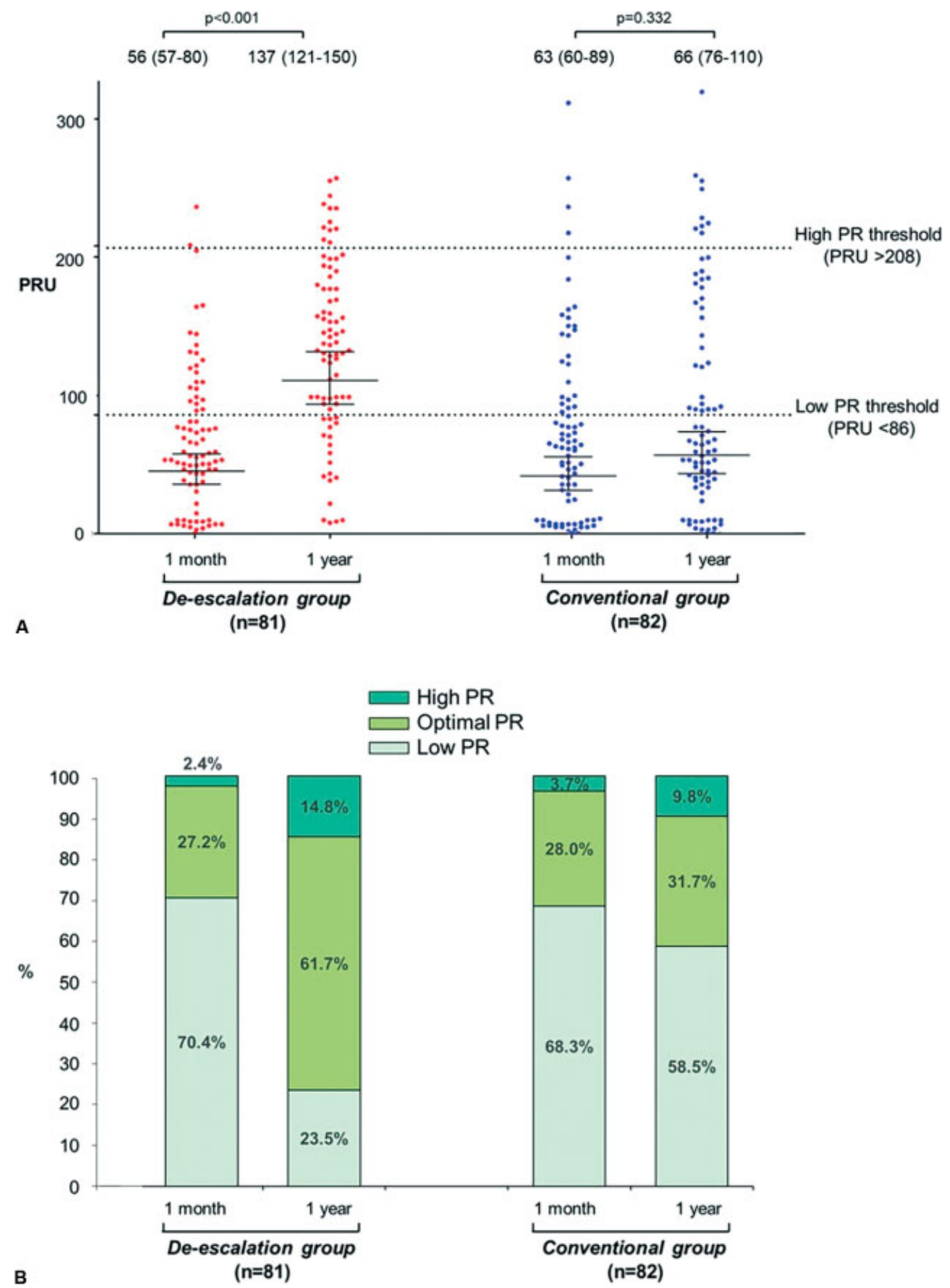

Fig. 1 Individual values of platelet reactivity and prevalence of low PR, optimal PR, and high PR by treatment sequence. (A) Plot of PRU values at 1-month and during 1-year follow-up in the de-escalation group versus the conventional group. PRU values are presented as median (95\% confidence interval). The line represents the median PRU and error bars represent $95 \%$ confidence interval. $p$-Values for the changes within each group are shown above the plots. (B) Prevalence of low PR, optimal PR, and high PR according to treatment sequence. The rate of optimal PR during 1-year follow-up was significantly higher in the de-escalation than in the conventional group. High PR, high on-treatment platelet reactivity; low PR, low on-treatment platelet reactivity; PRU, platelet reactivity unit.

during 1-year follow-up while on allocated therapy, the PRU value was significantly higher in the de-escalation group compared with the conventional group (137 [121-150] vs. 66 [76-110], $p<0.001$ ). The percentage of patients within the therapeutic range (OPR) was significantly higher in the deescalation compared with the conventional group (61.7 vs.
31.7\%, $p<0.001$ ) ( - Fig. 1B). Conversely, the percentage of patients with LPR was significantly higher in the conventional group ( 23.5 vs. $58.5 \%, p<0.001$ ) with more than half of the patients in the conventional group having LPR.

This prespecified analysis compared the prevalence of OPR between the de-escalation and conventional groups in the 
HOST-REDUCE-POLYTECH-ACS trial. Previous studies have shown an association between HPR and increased risk of stent thrombosis, and several observational studies have suggested a possible link between LPR and bleeding. ${ }^{5}$ Although it is unclear whether intermediate degree of PR (between the threshold for HPR and LPR), so-called "OPR" can lead to improved clinical outcomes, the possible benefit of OPR was suggested in a pooled analysis of several trials. ${ }^{6}$ The present pharmacodynamic analysis supports the favorable clinical outcomes seen in the de-escalation group over the conventional-dose group in the HOST-REDUCE-POLYTECH-ACS trial.

Some recent studies have suggested different therapeutic cutoffs to define HPR and LPR in East Asian patients. ${ }^{7}$ Using the proposed criteria of LPR of 127 from the A-MATCH trial in the present study, ${ }^{8}$ the number of patients with LPR would be 33 of $81(41 \%)$ in the de-escalation group and 59 of $82(72 \%)$ in the conventional group, again confirming that prasugrel $10 \mathrm{mg}$ would be too strong for East Asians.

First, our study is limited by the small sample size and lack of universally accepted cutoff values of OPR. In particular, although this analysis was preplanned, only six centers (out of 35) elected to participate in the PFT subanalysis and thus the decision to perform PFT was left up to the discretion of the participating investigators in the six centers, which could have resulted in selection bias. However, the number of patients measured for each allocated group and the baseline characteristics between the two groups were well balanced. Second, the dose of prasugrel used in the de-escalation arm $(5 \mathrm{mg})$ is not a dose that is universally approved for ACS patients. Further, the study was performed in a dedicated East Asian population. Both of these factors may limit the wide-spread direct applicability of our results. Third, we could not link the pharmacodynamic data directly with clinical outcomes due to the small number of patients tested and the low event rates in the original study. Therefore, further studies will need to prove whether OPR is associated with significantly reduced net adverse clinical events. In conclusion, de-escalation as compared with maintaining the conventional dose of prasugrel may result in a higher percentage of patients achieving the so-called "OPR" in ACS patients receiving $\mathrm{PCI}$. The findings are hypothesis-generating at best and require additional studies to confirm whether such concept is clinically relevant.

Funding

The authors received funding from Daiichi Sankyo, Boston Scientific, Terumo, Biotronik, Qualitech Korea, and Dio.
This study was supported by SNUH (Grant no. 06-20101090)

Conflict of Interest

H-.S.K. has received research grants or speaker's fees from Daiichi Sankyo, Boston Scientific, Terumo, Biotronik, and Dio, as well as Medtronic, Abbott Vascular, Edwards Life Science, AmGen, and Boehringer Ingelheim. K.W.P. reports fees from Daiichi Sankyo, Astra-Zeneca, Sanofi, BristolMyers Squibb, Bayer AG, and Pfizer outside of the submitted work. All other authors declare no competing interests.

\section{References}

1 Collet JP, Thiele H. The 'Ten Commandments' for the 2020 ESC Guidelines for the management of acute coronary syndromes in patients presenting without persistent ST-segment elevation. Eur Heart J 2020;41(37):3495-3497

2 Sibbing D, Aradi D, Alexopoulos D, et al. Updated expert consensus statement on platelet function and genetic testing for guiding $\mathrm{P}_{2} \mathrm{Y}_{12}$ receptor inhibitor treatment in percutaneous coronary intervention. JACC Cardiovasc Interv 2019;12(16): 1521-1537

3 Kim HS, Kang J, Hwang D, et al; HOST-REDUCE-POLYTECH-ACS investigators. Prasugrel-based de-escalation of dual antiplatelet therapy after percutaneous coronary intervention in patients with acute coronary syndrome (HOST-REDUCE-POLYTECHACS): an open-label, multicentre, non-inferiority randomised trial. Lancet 2020;396(10257):1079-1089

4 Campo G, Parrinello G, Ferraresi P, et al. Prospective evaluation of on-clopidogrel platelet reactivity over time in patients treated with percutaneous coronary intervention relationship with gene polymorphisms and clinical outcome. J Am Coll Cardiol 2011;57 (25):2474-2483

5 Tantry US, Bonello L, Aradi D, et al; Working Group on OnTreatment Platelet Reactivity. Consensus and update on the definition of on-treatment platelet reactivity to adenosine diphosphate associated with ischemia and bleeding. J Am Coll Cardiol 2013;62(24):2261-2273

6 Aradi D, Kirtane A, Bonello L, et al. Bleeding and stent thrombosis on P2Y12-inhibitors: collaborative analysis on the role of platelet reactivity for risk stratification after percutaneous coronary intervention. Eur Heart J 2015;36(27):1762-1771

7 Kim HK, Tantry US, Smith SC Jr, et al. The East Asian paradox: an updated position statement on the challenges to the current antithrombotic strategy in patients with cardiovascular disease. Thromb Haemost 2020;121(04):422-432

8 Jeong $\mathrm{YH}, \mathrm{Oh} \mathrm{JH}$, Yoon $\mathrm{HJ}$, et al. Pharmacodynamic profile and prevalence of bleeding episode in East Asian patients with acute coronary syndromes treated with prasugrel standard-dose versus de-escalation strategy: a randomized A-MATCH trial. Thromb Haemost 2021. Doi: 10.1055/a-1346-3300 